\title{
Lattice dynamics of 3-dimensional tilings modelling icosahedral quasicrystals
}

\author{
J. Los and T. Janssen \\ Institute for Theoretical Physics, Unitersty of Nijmegen, 6525 ED Nijmegen, The Netherlands \\ F. Gähler \\ Département de Phystque Théorique, Universtié de Genè̀e. 24, quai Ernest Ansermet, CH.I2II Genèce, Sw'tzerland
}

\begin{abstract}
A study of the lattice dynamics of three-dimensional tilings modelling icosahedral quasicrystals is presented. The phonon density of states is calculated, and the character of the eigenstates is determined. Three different types of commensurate approximants are considered, namely symmetrized, perfect and randomized approximants. It appears that the density of states is smoothed by randomization. The participation ratio. which measures the rate of localization of an eigenmode, is given as a function of frequency. Only the states at the very upper end of the frequency spectrum appear to be localized. whereas all other states are extended. The density of states at low frequencies is analyzed in more detail, by applying a Brillouin zone integration over the lowest branches. It is found that these lowest branches scale for successive approximants.
\end{abstract}

\section{Introduction}

Due to the lack of periodicity of quasicrystals, the calculation of physical properties, such as phonons and electronic structure, is more difficult than for ordinary crystals. Until now most research has been devoted to one-dimensional models, such as the Fibonacci chain. It has been shown for the Fibonacci chain and similar systems that their electron or phonon spectrum shows scaling behaviour $[1,2]$. The eigenstates in these systems are neither extended nor localized, but critical [3]. It remains to be seen, however, how much of this is still true for $3 \mathrm{~d}$ quasicrystals.

It is the purpose of this paper to shed some more light on these questions. In a previous paper [4], the phonon density of states (DOS) had been obtained for models based on the icosahedral Penrose tiling (i-PT). In the present study we

Correspondence to: Dr J. Los, Institute for Theoretical Physics, University of Nigmegen, 6525 ED Nijmegen. The Netherlands. consider, in addition, the DOS for structures based on icosahedral random tilings [5], which form another popular model for icosahedral quasicrystals [6]. Furthermore, for both kinds of models the eigenmodes of vibration are obtained and analyzed in terms of the inverse participation ratio, which is a measure for the degree of localization of an eigenmode.

The remainder of the paper is organized as follows. After introducing, in section 2, the different models to be studied, we present in section 3 their DOS and analyze the character of the eigenmodes. The results for the different models are compared. Section 4 is devoted to a detailed study of the low frequency bchaviour for struc. tures based on the Penrose pattern. We then conclude in section 5 .

\section{Structure and dynamical model}

The i-PT is the intersection of a periodic structure in 6 dimensions with 3D physical space. It is 
a quasiperiodic space filling by two types of tiles, a thick and a thin rhombohedron. By slightly deforming the six-dimensional structure relative to physical space one can construct tilings which are periodic approximants of the i-PT. Each approximant is a member of an infinite series of successive approximants [7]. In this paper, we consider approximants with a cubic or tetragonal unit cell. Cubic approximants are commonly denoted by the rational approximation to $\phi=(\sqrt{5}$ $+1) / 2$ which has been used in the deformation. Approximant tilings in general have no point group symmetry, but one can enforce tetrahedral symmetry (of order 24) by adding a few further sites to the structure. We shall call such structures symmetrized tilings.

Random tilings (see, e.g., ref. [6]) are obtained by applying a randomization procedure [5] to commensurate approximants. For this reason, we often call them randomized tilings. The randomization procedure consists of repeatedly flipping certain configurations of rhombohedra and preserves the periodicity. For details, we refer to Tang [5]. In this paper, we consider only maximally randomized tilings.

A dynamical model is obtained by placing atoms of unit mass on the vertices of the tiling, and connecting neighboring atoms by springs. In non-symmetrized and randomized approximants atoms are considered as neighbors if they have a distance corresponding to the short body diagonal of the thin rhombohedron, or to an edge or a short face diagonal of a tile. In the case of symmetrized tilings, one further short distance appears. Although we have experimented with different values of the spring constants we will choose all spring constants equal to one in this paper. The results for different spring constants are not substantially different. The dynamical matrix of this vibrating system of masses and springs is calculated in harmonic approximation and diagonalized numerically, using Born-von Karmann boundary conditions. Due to computational reasons, we often have to restrict ourselves to $k$-values yielding a real dynamical matrix. In the case of symmetrized tilings, the tetragonal symmetry is used to reduce the dynamical matrix to block diagonal form.

\section{Density of states and character of eigenmodes}

The density of states (DOS) $D(\omega)$ is defined such that $D(\omega) d \omega$ is the number of states between $\omega$ and $\omega+d \omega$. We shall use a smoothed DOS, especially useful when only a limited number of states is available, which is given by

$D(\omega)=\frac{1}{\pi} \operatorname{Im} \sum \frac{1}{\omega-\omega_{1}-i \epsilon}$,

where the sum runs over all states, and $c$ is a small positive number.

For the symmetrized $5 / 3$ approximant we have calculated all states belonging to $k$-points yielding real dynamical matrices. The result is shown in fig. 1, together with the DOS for an foc structure. The picture shows that the DOS is less pronounced for the $5 / 3$ approximant. For both curves, there is a global $\omega^{2}$ dependence at low frequencies, but the proportionality constant is somewhat lower in the $5 / 3$ case, which is a consequence of the difference in the mean number of neighbours. The low frequency region will be discussed in more detail in section 4.

To study the effect of randomization on the DOS we have taken a tetragonal approximant with 932 atoms in the unit cell and plotted the DOS for the perfect (non-randomized) structure against the DOS for two different randomizations

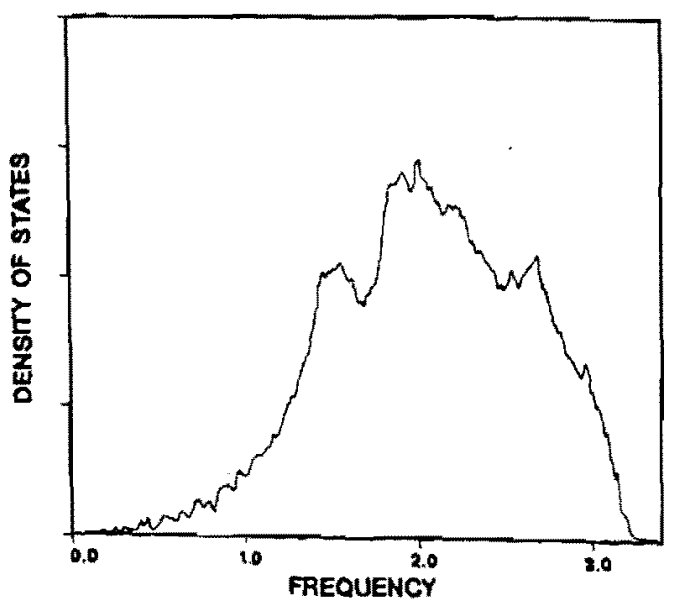

Fig. 1. The nomalized density of states for the symmetrized $5 / 3$ approximant (solid curve, 60720 states) and an (cc struc. ture (datted curve, 49152 states). Both curves are calculated with $\varepsilon=0.05$. 
(fig. 2). In all three cases only the states for $k$-points yielding a real dynamical matrix were included. The result for the non-randomized case is similar to that for the $5 / 3$ approximant, but in the randomized cases the first peak completely disappears. There is hardly any difference between the two randomized cases, which suggests that the two samples are typical randomized tilings. At low frequencies there is no difference between the randomized and non-randomized cases. Obviously, the accoustic modes are not affected by the fine structure of the tilings.

The character of the eigenvectors is analyzed by means of the inverse participation ratio (IPR), given by $\Sigma_{t}\left|\boldsymbol{u}_{t}\right|^{4}$, where $\boldsymbol{u}_{1}$ is the displacement of the $i$ th atom in a normalized eigenmode (i.e. $\Sigma_{1}\left|u_{1}\right|^{2}=1$ ). The IPR measures the degree of localization of an eigenmode: high IPR correspond to localized states whereas low IPR corresponds to extended states. In fig. 3 the IPR is shown as a function of frequency for the symmetrized 5/3 approximant. The results for clusters and randomized tilings are all similar to fig. 3 , showing that in all cases only the highest frequency states are localized. The IPR shows some more structure for the symmetrized tilings, which is probably due to the larger variation in the number of neighbors. Randomization reduces the

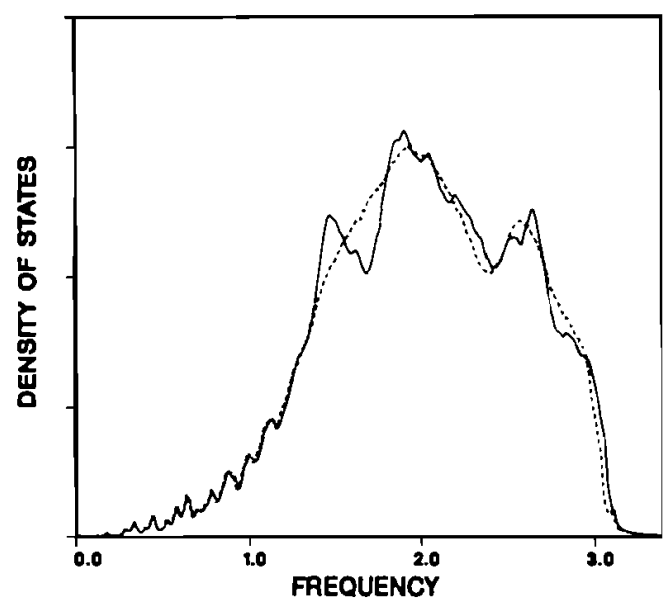

Fig. 2. The density of states for a perfect approxımant with 932 atoms (solid curve) and two randomizations (dotted and dashed curves).

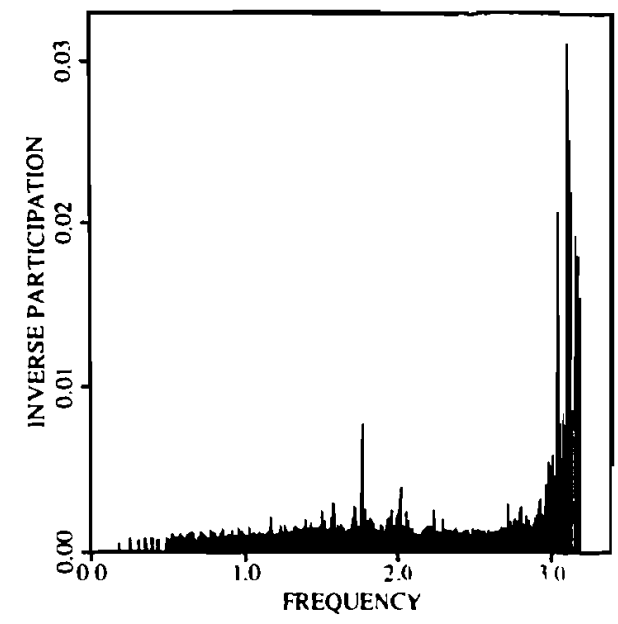

Fig. 3. The inverse participation ratios for the symmetrized $5 / 3$ approximant. Al states for $k$-values yielding real dynamlcal matrices are included.

variation in the connectivity, making the tilings more homogeneous, which might explain why the inverse participation function for randomized tilings is still smoother than that for non-randomized ones, which is opposite to what one might have expected.

\section{Scaling properties at low frequencies}

Due to the lack of translational symmetry it is difficult to find the detailed structure of the DOS for the i-PT directly, even at low frequencies. One can, however, do calculations for successive commensurate approximants and then, by using scaling arguments, predict the results for the infinite i-PT.

For successive approximants we determined the lowest 20 eigenfrequencies on a cubic grid in the Brillouin zone, and the density of states was calculated by integrating over the lowest 20 branches, using linear interpolation. In grid- $n$ ap. proximation, an octant of the cubic Brillouin zone is devided into $n^{3}$ small cubes. The eigenvalues are calculated for all $k$-points on the corners of those cubes. We have chosen cubic symmetrized approximants, which reduces the irreducible part of the Brillouin zone by a factor 12. Still, for 

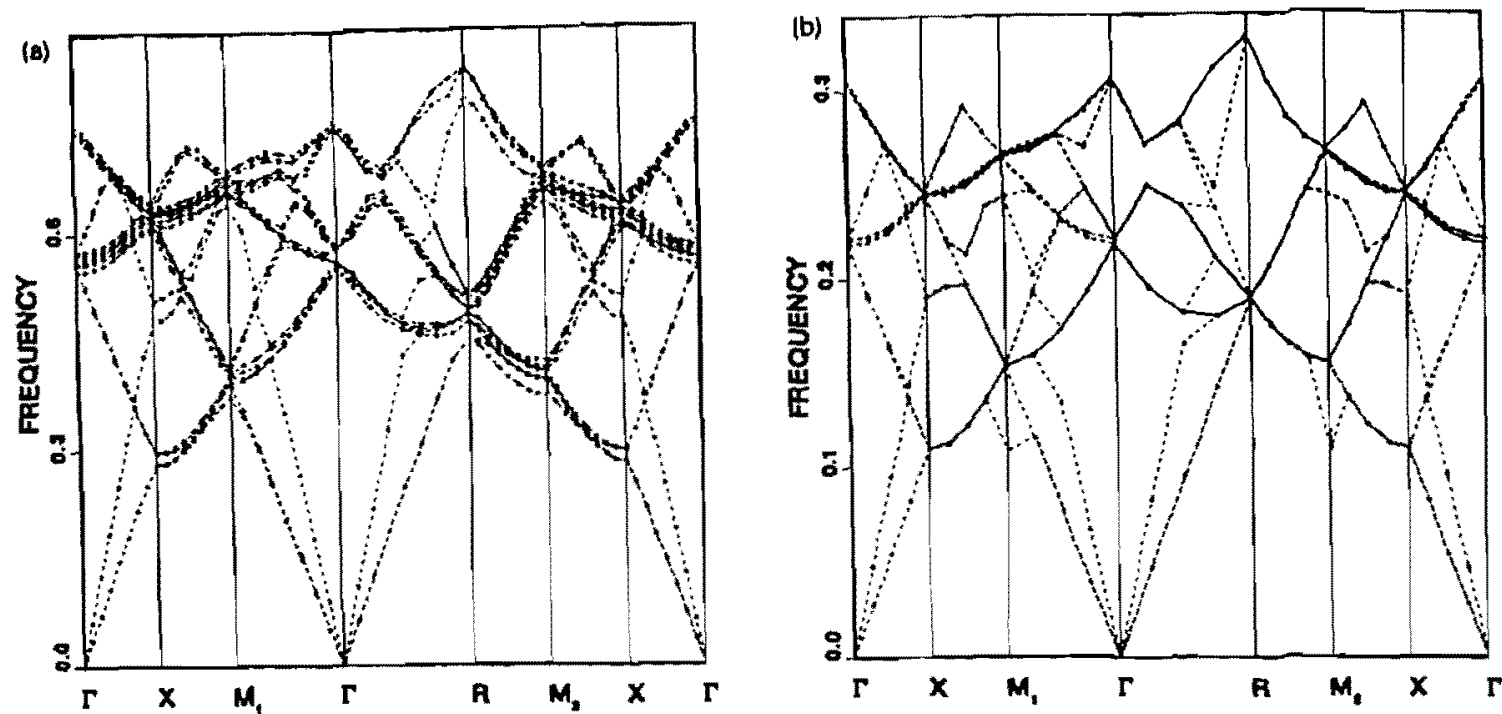

Fig. 4. The 20 lowest branches along a path in the Brillouin zone tor the $3 / 2$ approximant (a) and the $8 / 5$ approximant (b). $\Gamma=(0,0,0), X=(\pi / a)(1,0,0), M_{1}=(\pi / a)(1,1,0), R=(\pi / a)(1,1,1), M_{2}=(\pi / a)(1,0,1)$.

arbitrary $k$-vector the dynamical matrix has no symmetry and becomes very large for the higher approximants. However, the dynamical matrices are sparse, and we took advantage of this by applying a Lanczos algorithm without reorthogonalization $[8]$ to find the required number of lowest eigenvalues.

The scaling behavior of the spectrum is studied by plotting the lowest 20 branches along a path in the Brillouin zone. Figure 4(a) shows the results for a $3 / 2$ approximant, and fig. $4(\mathrm{~b})$ those for an $8 / 5$ approximant. Although band crossings will occur along this path due to the symmetry, the branches will not cross in the interior of the Brillouin zone, and therefore we have connected the frequencies in ascending order. Even though symmetrization makes scaling for successive approximants less complete, these pictures clearly show that the spectrum scales.

The DOS resulting from the lowest 20 branches is shown in fig. 5 , for the $3 / 2$ approximant in grid-8 approximation, and for the $8 / 5$ approximant in grid -4 approximation. For the latter also the eigenvalues at the centres of the grid cubes were used. Experience with a 2/1 approximant shows that going beyond grid-8 approximation does not change much. If this is true also for the
$3 / 2$ approximant we must conclude that there is deviation from the normal $\omega^{2}$ behaviour already at relatively low frequencies. Higher grid densities might weaken these deviations somewhat however.

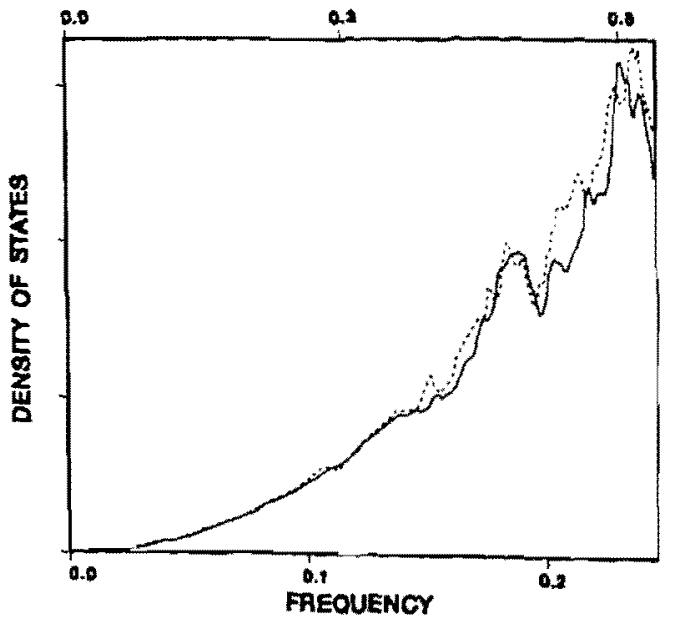

Fig. 5. The density of states resulting from the lowest 20 branches for the $3 / 2$ approximant (dashed) and the $8 / 5$ approximant (solid line). The labelling on the upper $x$-axis is for the $3 / 2$ approximant. 


\section{Conclusion}

The phonon density of states and the vibration eigenmodes have been studied for structures based on i-PTs and randomized tilings. While our models are probably not realistic enough to be compared directly to experiments, we nevertheless expect that they show the essential features of realistic structures.

Our randomization program is partly based on code written by L.H. Tang. We thank him for freely sharing his code with us.

\section{References}

[1] M. Kohmoto. L.P. Kadanoff and Chav Tang, Phys. Rev. LeII. $50(1983) 1870$.

[2] G. Gumbs and M.K. Ali. Phys. Rev. Lell $(6)(19 \times 8)$ 1081

[3] J.M. Luck and D. Petritis, J. Stat. Phys. 4211986$) 284$.

[4] 1. Los and T. Janssen. 1. Phys: Condens. Maller 2 (1990) 9553.

[5] L.H. Tang, Phys. Rev. Lett, 64 (1990) 2304).

[6] C.L. Henley. Random tiling models, in: Quasucrystals: The State of the Art, eds. P.J. Stemhardi and D.P. DiVincenco (World Scientific. Singapore, 1991).

[7] T. Janssen. Europhys. Let. 14 (1991) 131.

[8] J.K. Cullum and R.A. Willoughby. Lanczos Algorithms for Large Symmetric Eigenvalue Computation (Brkhiuser. Basle, 1985). 